\title{
Molecular Clouds in the LMC Observed with NANTEN I. Physical Properties of the Clouds
}

\author{
N. Mizuno, R. Abe, A. Hara, T. Hayakawa, S. Kato, A. Kawamura, \\ A. Mizuno, H. Ogawa, T. Onishi, H. Saito, K. Tachihara, K. C. Xiao, \\ N. Yamaguchi, R. Yamaguchi, and Y. Fukui \\ Department of Astrophysics, Nagoya University, Chikusa-ku, Nagoya, \\ 464-8602, Japan
}

\section{Y. Yonekura}

Earth and Life Sciences, Osaka Prefecture University, Gakuencho 1-1, Sakai, Osaka 599-8531, Japan

\begin{abstract}
Fully sampled ${ }^{12} \mathrm{CO}(J=1-0)$ observations of the whole extent of the LMC have been made with a linear resolution of $\sim 30 \mathrm{pc}$ at a detection limit of $N\left(\mathrm{H}_{2}\right)=2 \times 10^{21} \mathrm{~cm}^{-2}$. In addition, several selected regions have been mapped with higher sensitivity corresponding to a detection limit of $1 \times 10^{21} \mathrm{~cm}^{-2}$. Based on these results, a new estimate of the molecular mass in the LMC is presented.
\end{abstract}

\section{Observations}

A ${ }^{12} \mathrm{CO}(J=1-0)$ survey of the LMC was made in the period between 1997 February and November, using the 4 meter millimeter-wave telescope NANTEN of Nagoya University located at the Las Campanas Observatory, Chile. The $4 \mathrm{~K}$ cooled SIS mixer receiver provided a typical single sideband (SSB) system temperature of $210 \mathrm{~K}$ toward the zenith including the atmosphere. The spectrometer was an acousto-optical spectrometer (AOS) which covers a velocity range of 100 $\mathrm{km} \mathrm{s}^{-1}$ and has a velocity resolution of $0.1 \mathrm{~km} \mathrm{~s}^{-1}$. All the observations were performed using the position-switching technique. The integration time at each position was about 1 minute and the rms noise fluctuations were around $0.35 \mathrm{~K}$ at a velocity resolution of $0.1 \mathrm{~km} \mathrm{~s}^{-1}$.

Subsequently, to reveal the distribution of faint molecular gas, additional more sensitive observations were made from 1998 April through June, at the rms noise level of $0.16 \mathrm{~K}$, in the same line at the same angular resolution for several selected regions including 30 Dor, the southern large CO complex, N11, N44, and the Bar.

\section{Molecular Cloud Properties}

A fully sampled CO map of the LMC and the general properties of giant molecular clouds are presented by Fukui et al. (1998). These observations have been made at a grid spacing of $2^{\prime}(\sim 30 \mathrm{pc})$ with a $2^{\prime} \cdot 6$ beam. In the observed area 

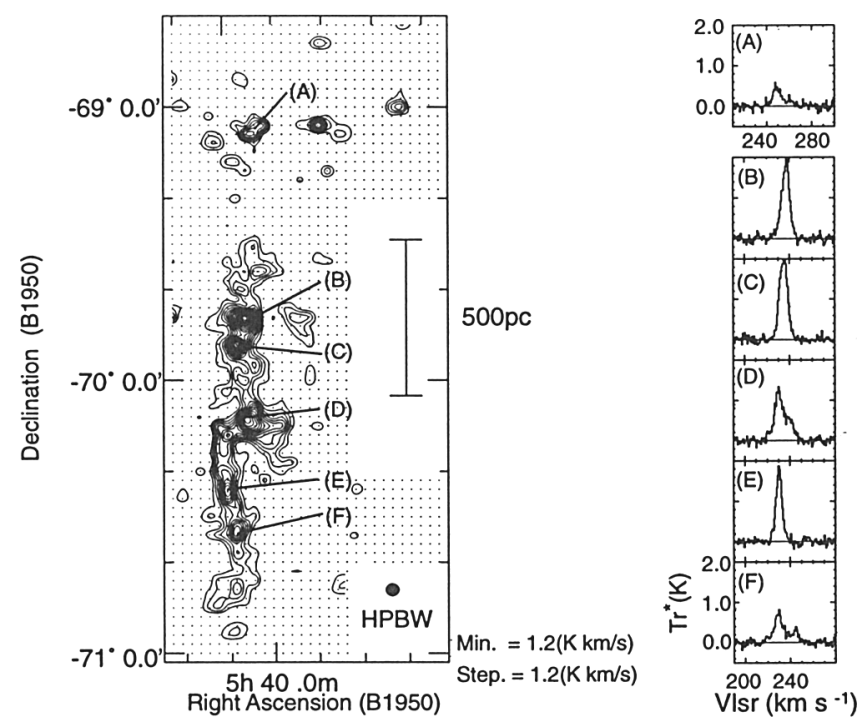

Figure 1. A contour map of the integrated ${ }^{12} \mathrm{CO}(J=1-0)$ emission in 30 Doradus and the southern large CO complex. The spectra of six major peak positions of clouds are also shown.

we identified 55 molecular clouds which are detected at more than 3 observed points having a ${ }^{12} \mathrm{CO}$ integrated intensity greater than the $4 \sigma$ noise level $(2.4$ $\mathrm{K} \mathrm{km} \mathrm{s}^{-1}$ ). The $\mathrm{CO}$ clouds have radii ranging from 30 to $100 \mathrm{pc}$, line widths between 3.5 and $13 \mathrm{~km} \mathrm{~s}^{-1}$, CO luminosities between $8 \times 10^{3}$ and $2 \times 10^{5} \mathrm{~K} \mathrm{~km}$ $\mathrm{s}^{-1} \mathrm{pc}^{2}$, and virial masses ranging from $6 \times 10^{4}$ to $2 \times 10^{6} \mathrm{M}_{\odot}$.

In Figure 1, we present the map as well as several sample spectra from the deep observations. The peak intensities are $T_{r}^{*}=2 \mathrm{~K}$ in Figure 1 , while the typical ${ }^{12} \mathrm{CO}$ spectrum in the LMC has a peak intensity of $T_{\mathrm{r}}^{*} \lesssim 1 \mathrm{~K}$.

According to these deep observations, the masses of the large $\mathrm{CO}$ clouds, which were detected at more than 3 observed points in the first observations, increase by $120 \%$, and the masses of the smaller CO clouds increase by $160 \%$. We also detected some new, small clouds whose mass range from $10^{4}$ to $10^{5} \mathrm{M}_{\odot}$. From these results, we estimate that the total molecular mass may increase up to $\sim 7 \times 10^{7} \mathrm{M}_{\odot}$, about twice as large as our previous estimate, $\sim 4 \times 10^{7} \mathrm{M}_{\odot}$, if the deep observations were made over the whole galaxy.

We also identified clouds at the $3 \sigma$ and the $6 \sigma$ noise level, in order to test how much the conversion factor, $X$, depends upon the cloud definition. We find that the $X$ factor is independent of cloud definition for a sample of $20 \mathrm{GMCs}$.

Acknowledgments. We appreciate the hospitality of all the staff members of the Las Campanas Observatory of the Carnegie Institution of Washington.

\section{References}

Fukui et al. 1999, this volume 